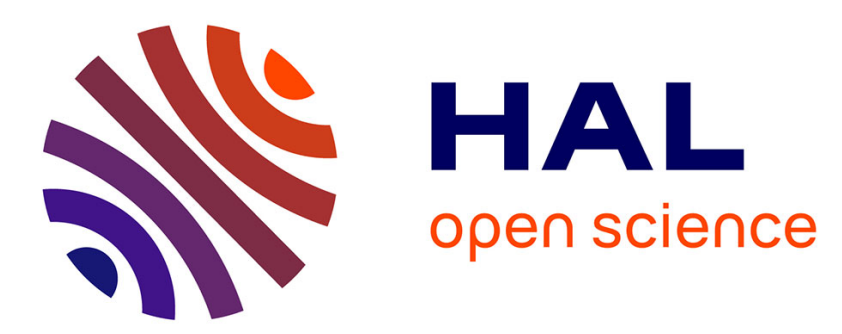

\title{
Image-based visual servo control of the translation kinematics of a quadrotor aerial vehicle
}

O. Bourquardez, R. Mahony, N. Guenard, François Chaumette, Tarek Hamel, L. Eck

\section{- To cite this version:}

O. Bourquardez, R. Mahony, N. Guenard, François Chaumette, Tarek Hamel, et al.. Image-based visual servo control of the translation kinematics of a quadrotor aerial vehicle. IEEE Transactions on Robotics, 2009, 25 (3), pp.743-749. inria-00436722

\section{HAL Id: inria-00436722 \\ https://hal.inria.fr/inria-00436722}

Submitted on 27 Nov 2009

HAL is a multi-disciplinary open access archive for the deposit and dissemination of scientific research documents, whether they are published or not. The documents may come from teaching and research institutions in France or abroad, or from public or private research centers.
L'archive ouverte pluridisciplinaire HAL, est destinée au dépôt et à la diffusion de documents scientifiques de niveau recherche, publiés ou non, émanant des établissements d'enseignement et de recherche français ou étrangers, des laboratoires publics ou privés. 
[3] Y. Kawauchi, I. Makoto, and T. Fukuda, "A principle of distributed decision making of cellular robotic system (CEBOT)," in Proc. IEEE Int. Conf. Robot. Autom., Piscataway, NJ: IEEE Press, 1993, pp. 833-838.

[4] M. Yim, K. Roufas, D. Duff, Y. Zhang, C. Eldershaw, and S. B. Homans, "Modular reconfigurable robots in space applications," Auton. Robots, vol. 14, no. 2/3, pp. 225-237, 2003.

[5] M. Yim, D. G. Duff, and K. D. Roufas, "PolyBot: A modular reconfigurable robot," in Proc. IEEE Int. Conf. Robot. Autom., vol. 1, Piscataway, NJ: IEEE Press, 2000, pp. 514-520.

[6] A. Castano, W. M. Shen, and P. Will, "CONRO: Towards deployable robots with inter-robots metamorphic capabilities," Auton. Robots, vol. 8, no. 3, pp. 309-324, 2000.

[7] S. Hirose, T. Shirasu, and E. F. Fukushima, "Proposal for cooperative robot "Gunryu" composed of autonomous segments," Robot. Auton. Syst., vol. 17, pp. 107-118, 1996.

[8] H. B. Brown, J. M. V. Weghe, C. A. Bererton, and P. K. Khosla, "Millibot trains for enhanced mobility," IEEE/ASME Trans. Mechatron., vol. 7, no. 4, pp. 452-461, Dec. 2002.

[9] R. Damoto, A. Kawakami, and S. Hirose, "Study of super-mechano colony: Concept and basic experimental set-up," Adv. Robot., vol. 15, no. 4, pp. 391-408, 2001.

[10] K. Motomura, A. Kawakami, and S. Hirose, "Development of arm equipped single wheel rover: Effective arm-posture-based steering method," Auton. Robots, vol. 18, no. 2, pp. 215-229, 2005.

[11] S. Murata, E. Yoshida, A. Kamimura, H. Kurokawa, K. Tomita, and S. Kokaji, "M-tran: Self-reconfigurable modular robotic system," IEEE/ASME Trans. Mechatron., vol. 7, no. 4, pp. 431-441, Dec. 2002

[12] W. M. Shen, M. Krivokon, H. Chiu, J. Everist, M. Rubenstein, and J. Venkatesh, "Multimode locomotion for reconfigurable robots," Auton. Robots, vol. 20, no. 2, pp. 165-177, 2006.

[13] M. Yim, W. M. Shen, B. Salemi, D. Rus, M. Moll, H. Lipson, E. Klavins, and G. S. Chirikjian, "Modular self-reconfigurable robot systems," IEEE Robot. Autom. Mag., vol. 14, no. 1, pp. 43-52, Mar. 2007.

[14] D. Rus and M. Vona, "Crystalline robots: Self-reconfiguration with compressible unit modules," Auton. Robots, vol. 10, no. 1, pp. 107-124, 2001.

[15] P. White, V. Zykov, J. Bongard, and H. Lipson, "Three dimensional stochastic reconfiguration of modular robots," in Proc. Robot. Sci. Syst., Cambridge, MA: MIT Press, 2005, pp. 161-168.

[16] C. Jones and M. J. Matarić, "From local to global behavior in intelligent self-assembly," in Proc. IEEE Int. Conf. Robot. Autom., vol. 1, Los Alamitos, CA: IEEE Comput. Soc. Press, 2003, pp. 721-726.

[17] E. Klavins, R. Ghrist, and D. Lipsky, "A grammatical approach to selforganizing robotic systems," IEEE Trans. Autom. Control, vol. 51, no. 6, pp. 949-962, Jun. 2006.

[18] W. M. Shen, P. Will, A. Galstyan, and C. M. Chuong, "Hormone-inspired self-organization and distributed control of robotic swarms," Auton. Robots, vol. 17, no. 1, pp. 93-105, 2004.

[19] A. L. Christensen, R. O'Grady, and M. Dorigo, "Morphology control in a multirobot system," IEEE Robot. Autom. Mag., vol. 14, no. 4, pp. 18-25, Dec. 2007.

[20] F. Mondada, L. M. Gambardella, D. Floreano, S. Nolfi, J.-L. Deneubourg, and M. Dorigo, "The cooperation of swarm-bots: Physical interactions in collective robotics," IEEE Robot. Autom. Mag., vol. 12, no. 2, pp. 21-28, Jun. 2005

[21] A. L. Christensen, "Efficient neuro-evolution of hole-avoidance and phototaxis for a swarm-bot,” D.E.A. thesis, Inst. Recherches Interdisciplinaires Dév. Intell. Artif. (IRIDIA), Univ. Libre de Bruxelles, Brussels, Belgium, Tech. Rep. TR/IRIDIA/2005-14, Oct. 2005.

\section{Image-Based Visual Servo Control of the Translation Kinematics of a Quadrotor Aerial Vehicle}

\author{
Odile Bourquardez, Robert Mahony, Nicolas Guenard,
} François Chaumette, Tarek Hamel, and Laurent Eck

\begin{abstract}
In this paper, we investigate a range of image-based visual servo control algorithms for regulation of the position of a quadrotor aerial vehicle. The most promising control algorithms have been successfully implemented on an autonomous aerial vehicle and demonstrate excellent performance.
\end{abstract}

Index Terms-Aerial robotic vehicle, visual servoing.

\section{INTRODUCTION}

Visual servo algorithms have been extensively developed in the robotics field over the last ten years [7], [10], [19], [23]. Visual servo control techniques have also been applied recently to a large variety of reduced-scale aerial vehicles, such as quadrotors [1], [25], helicopters [2], [22], [26], [29], airships [4], [30], and airplanes [5], [24]. In this paper, we consider visual servo control of a quadrotor aerial vehicle.

Much of the existing research in visual servo control of aerial robots (and particularly, autonomous helicopters) has used position-based visual servo techniques [1], [2], [22], [25]-[27], [29]. The estimated pose can be used directly in the control law [1], or as part of a scheme fusing visual data and inertial measurements [29]. In this paper, we do not deal with pose estimation, but consider image-based visual servo (IBVS), similar to the approach considered in [4], [17], and [30].

The system dynamics is sometimes explicitly taken into account in IBVS. This strategy has been applied for robotic manipulators [9], [12], [20] and for aerial vehicles [15], [30]. Another popular approach (as usually done for most robotic systems such as robot arms, mobile robots, etc.) is based on separating the control problem into an inner loop and an outer position control loop. As for helicopters, the inner attitude loop is run at high gain using inputs from inertial sensors, rate gyrometers, and accelerometers acquired at high data rate, while the outer loop is run at low gain using video input from the camera [26], [27]. The outer (visual servo) loop provides set points for the inner attitude loop and classical time-scale separation and high-gain arguments can be used to ensure stability of the closed-loop system [1], [11], [15], [27].

Manuscript received June 17, 2008; revised December 4, 2008. First published February 2, 2009; current version published June 5, 2009. This paper was recommended for publication by Associate Editor P. Rives and Editor W. K. Chung upon evaluation of the reviewers' comments. This work was supported by the Centre National de la Recherche Scientifique (CNRS) under the Project Robotique et Entités Artificielles (ROBEA)-Robvolint and the International Programs for Scientific Cooperation (PICS) between France and Australia on visual servo-control of unmanned aerial vehicles.

O. Bourquardez and F. Chaumette are with the Institut de Recherche en Informatique et Systèmes Aléatoires (IRISA)-Centre National de la Recherche Scientifique (CNRS) and l'Institut National de Recherche en Informatique et en Automatique (INRIA), 35042 Rennes, France (e-mail: odile.bourquardez@ voila.fr; francois.chaumette@irisa.fr).

R. Mahony is with the Department of Engineering, Australian National University, Canberra, A.C.T. 0200, Australia (e-mail: robert.mahony@anu.edu.au).

N. Guenard and L. Eck are with the Commissariat à l'Energie Atomique (CEA)/List, 92265 Fontenay-aux-Roses, France (e-mail: nicolas.guenard@ cea.fr; laurent.eck@cea.fr).

T. Hamel is with the Laboratoire d'Informatique, Signaux et Systèmes de Sophia antipolis (I3S), Université de Nice Sophia-Antipolis (UNSA)-Centre National de la Recherche Scientifique (CNRS), 06903 Sophia Antipolis, France (e-mail: thamel@i3s.unice.fr).

Color versions of one or more of the figures in this paper are available online at http://ieeexplore.ieee.org.

Digital Object Identifier 10.1109/TRO.2008.2011419 
In this paper, we take the inner/outer loop stability for granted (see [14] for details) and concentrate on the specific properties of the outer IBVS control design. It allows designing kinematic controllers, which give many advantages in practice. For example, using an embedded camera that sends the images to a ground station implies time delays and then a slow image-based control loop. It is thus interesting to have a lower level loop to ensure the stabilization of the system. Then, another advantage to consider kinematic control is to enable easier reuse of the IBVS scheme, since it is not close to the material equipment of the aerial vehicle. In this paper, several control schemes are proposed, compared, and the most promising ones are shown to be stable in practice and to provide satisfactory behavior.

Following earlier work [15], [17], [28], we have chosen to use zero and first-order image moments as primary visual features for the control design. Perspective projection moments with suitable scaling along with a classical IBVS control design lead to satisfactory transients and asymptotic stability of the closed-loop system when the image plane remains parallel to a planar target. However, the system response may lack robustness for aggressive maneuvers. In order to overcome this problem, several control schemes, based on spherical first-order image moments, are designed and their performance is analyzed. The most promising control algorithms have been successfully implemented on an autonomous aerial vehicle showing excellent performance.

The paper is organized as follows. Section II develops a classical IBVS control scheme using perspective image moments. Section III introduces the definition and properties of first-order spherical image moments and presents a range of control laws for the translational motion of the camera using this visual feature. Section IV provides an analysis and a comparison of the control laws proposed. Experimental results are presented in Section V.

\section{Perspective Projection}

In this section, an IBVS control for regulation of the translation kinematics of an aerial vehicle is presented.

In order to obtain a quasi-linear and decoupled link between the image space and the task space, the image features used are perspective projection image moments [28]. The visual feature vector $\mathbf{s}=\left(x_{n}, y_{n}, a_{n}\right)$ is defined such that [28]

$$
a_{n}=Z^{*} \sqrt{\frac{a^{*}}{a}}, \quad x_{n}=a_{n} x_{g}, \quad y_{n}=a_{n} y_{g}
$$

where $a$ is the area of the object in the image, $x_{g}, y_{g}$ its centroid coordinates, $a^{*}$ the desired area, and $Z^{*}$ the desired depth between the camera and the target. The time derivative of $\mathbf{s}$ and the relative motion between the camera and the object can be related by the classical equation

$$
\dot{\mathbf{s}}=\mathbf{L}_{\boldsymbol{v}} \boldsymbol{v}+\mathbf{L}_{\boldsymbol{\omega}} \boldsymbol{\omega}
$$

where $\boldsymbol{v}$ and $\boldsymbol{\omega}$ are, respectively, the linear and angular velocity of the camera both expressed in the camera frame, and where $\mathbf{L}_{v}$ and $\mathbf{L}_{\boldsymbol{\omega}}$ are, respectively, the parts of the interaction matrix related to the translational and rotational motions. The desired image feature is denoted by $\mathbf{s}^{*}$, and the visual error is defined by $\mathbf{e}=\mathbf{s}-\mathbf{s}^{*}$.

Classical IBVS control design aims to impose linear exponential stability on the image error kinematics [10], [21], [28] to ensure an exponential decoupled decrease for $\mathbf{e}(\dot{\mathbf{e}}=-\lambda \mathbf{e}$, with $\lambda$ a positive gain). Using e to control the translational degrees of freedom, the classical IBVS control input is

$$
\boldsymbol{v}=-\left(\mathbf{L}_{\boldsymbol{v}}\right)^{-1}\left(\lambda \mathbf{e}+\mathbf{L}_{\boldsymbol{\omega}} \boldsymbol{\omega}\right), \quad \lambda>0 .
$$

Generally, the interaction terms $\mathbf{L}_{v}$ and $\mathbf{L}_{\boldsymbol{\omega}}$ depend nonlinearly on the state of the system and cannot be reconstructed exactly from the observed visual data. The visual feature $\mathbf{s}=\left(x_{n}, y_{n}, a_{n}\right)$ is of particular interest since $\mathbf{L}_{v}=-\mathbf{I}_{3}$ in the case where the camera image plane is parallel to the target plane [28]. In that case, since the link between image space and task space is linear and decoupled, the control scheme (2) is known to lead to satisfactory closed-loop behavior for holonomic robot [28]. It is, in fact, equivalent to a position-based visual servo, but without any pose estimation required.

In the application considered, the camera is mounted to point directly downward in the quadrotor and the image and target plane are never more than a couple of degrees offset. As a consequence, the approximation $\mathbf{L}_{\boldsymbol{v}} \approx-\mathbf{I}_{3}$ is valid. Furthermore, the motion of the quadrotor is smooth and slow and the value of $\mathbf{L}_{\boldsymbol{\omega}} \boldsymbol{\omega}$ is small compared with the error $\lambda \mathbf{e}$ in (2). Thus, a reasonable approximation of (2) for the purposes of this paper is

$$
\boldsymbol{v}=\lambda \mathbf{e}, \quad \lambda>0 .
$$

Equation (3) does not require the estimation of any 3-D parameters and can be implemented based only on the observed image features $\mathbf{s}$. This control was implemented on the experimental platform and the results are discussed in Section V-B. The limitation of this approach, however, lies in its dependence on the particular geometry of the application considered and the requirement to consider only smooth slow trajectories of the vehicle. If the vehicle undertakes aggressive manoeuvres, or the parallel target plane assumption is invalidated for a particular application, the approximation $\mathbf{L}_{\boldsymbol{v}} \approx-\mathbf{I}_{3}$ will fail, and more importantly, the approximation $\mathbf{L}_{\boldsymbol{\omega}} \boldsymbol{\omega} \approx \mathbf{0}$ may also fail. This second issue introduces a significant dynamic disturbance in the system response that cannot be cancelled directly without the risk of introducing zero dynamic effects into the closed-loop response similar to those studied in recent research [11], [18]. The potential limitations of the classical IBVS control design based on perspective projection features motivate us to consider a class of spherical projection features and nonlinear control design techniques.

\section{SPHERICAL PROJECTION}

\section{A. Modeling}

In this section, we use an unnormalized first-order spherical image moment along with an inertial goal vector to generate an image error [17]. Consider a point target consisting of $n$ points $\mathbf{P}_{i}$ corresponding to image points $\mathbf{p}_{i}(i \in(1, \ldots, n))$ on the spherical image surface. The centroid of a target is defined to be $\mathbf{q}=\sum_{i=1}^{n} \mathbf{p}_{i}$. The centroid $\mathbf{q}$ is a 3 -D vector. Thanks to the spherical camera geometry, the third entry of the centroid is nonlinearly related to the depth of the camera from the observed target constellation.

For a point target comprising a finite number of image points, the kinematics of the image centroid are easily verified to be [17] $\dot{\mathbf{q}}=$ $-\boldsymbol{\omega} \times \mathbf{q}-\mathbf{Q} \boldsymbol{v}$, where $\mathbf{Q}=\sum_{i=1}^{i=n} \frac{\boldsymbol{\pi}_{\mathbf{p}_{i}}}{\left|\mathbf{P}_{i}\right|}$ and $\boldsymbol{\pi}_{\mathbf{p}}=\left(\mathbf{I}_{3}-\mathbf{p} \mathbf{p}^{\top}\right)$. As long as there are at least two points $\mathbf{p}_{i}$ in image space, the matrix $\mathbf{Q}$ is positive definite [17].

Let $\mathbf{b}$ denote the vector that defines the direction of the fixed desired set point for the visual feature $\mathbf{q}$, expressed in a fixed inertial frame $\mathcal{F}_{A}$. The image-based error considered is

$$
\delta=\mathbf{q}-\mathbf{q}^{*}
$$

where $\mathbf{q}^{*}=\mathbf{R}^{\top} \mathbf{b}$, and the rotation matrix $\mathbf{R}$ between the camera frame $\mathcal{F}_{C}$ and the fixed inertial frame $\mathcal{F}_{A}$ (see Fig. 1) is assumed to be known, a common assumption when dealing with the control of under-actuated systems such as helicopters [17].

The reason for choosing the image error in this manner is that it ensures the passivity property. The image error kinematics are [17]

$$
\dot{\delta}=\delta \times \omega-\mathrm{Q} v .
$$




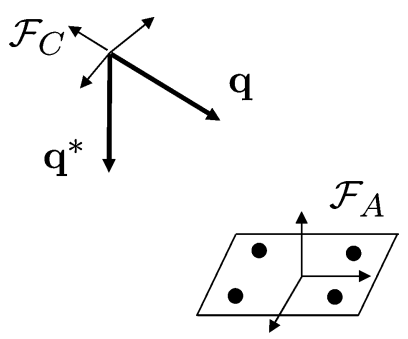

Fig. 1. Camera frame $\mathcal{F}_{C}$, fixed frame $\mathcal{F}_{A}$, and visual features $\mathbf{q}$ and $\mathbf{q}^{*}$.

It can be shown that $|\boldsymbol{\delta}|$ and $\boldsymbol{\delta}_{0}=\mathbf{R} \boldsymbol{\delta}$ are a function of position only [6]. This property can be exploited to control the translational dynamics independently of the rotations.

\section{B. Proportional Control}

A pure proportional feedback of the unnormalized centroid [17] ensures global asymptotic stability (GAS) property, but in practice it has been shown in [6] that task space and image space behavior are not acceptable. This is due to the fact that the convergence rates are given by $\mathbf{Q}$, and this matrix is not well-conditioned. The simple control law

$$
\boldsymbol{v}=k_{\delta} \boldsymbol{\delta}, \quad k_{\delta}>0
$$

is thus not suitable in practice.

\section{Partitioned Control}

A solution for compensating the poor sensitivity in the previous control design is to use a partitioned approach by singling out the problematic component for special treatment [8], [15].

The idea is to separate the visual error term into two criteria with different sensitivity. The new visual feature

$$
\boldsymbol{\delta}_{\mathbf{A}}=\boldsymbol{\delta}_{11}+\lambda \mathbf{q}_{0}^{*} \delta_{12}
$$

is defined by using the constant $\lambda$ (chosen as shown in [6]), and the following two new error terms

$$
\boldsymbol{\delta}_{11}=\mathbf{q}_{0}^{*} \times \mathbf{q}, \quad \delta_{12}=\mathbf{q}_{0}^{* \top} \boldsymbol{\delta}, \quad \text { with } \mathbf{q}_{0}^{*}=\frac{\mathbf{q}^{*}}{\left|\mathbf{q}^{*}\right|} .
$$

It can be shown that the control law

$$
\boldsymbol{v}=k_{\mathbf{A}} \mathbf{A}\left(\mathbf{q}_{0}^{*}\right)^{\top} \boldsymbol{\delta}_{\mathbf{A}}, \quad k_{\mathbf{A}}>0
$$

with $\mathbf{A}\left(\mathbf{q}_{0}^{*}\right)=\operatorname{sk}\left(\mathbf{q}_{0}^{*}\right)+\lambda \mathbf{q}_{0}^{*} \mathbf{q}_{0}^{* \top}$ ensures that the system is GAS [6]. Note that $\operatorname{sk}\left(\mathbf{q}_{0}^{*}\right)$ is the skew-symmetric matrix such that $\operatorname{sk}\left(\mathbf{q}_{0}^{*}\right) \mathbf{w}=$ $\mathbf{q}_{0}^{*} \times \mathbf{w}$ for any vector $\mathbf{w}$.

This partitioned control scheme has been used in [15] by designing and experimenting a dynamic control of a quadrotor. As shown in Section V-C1, although it enables to ensure the desirable GAS property in practice, the partitioned control scheme can lead to poor behavior of the system as soon as the distance between initial and desired position increases [6], [15]. In order to ensure good behavior in practice, we propose the following control laws.

\section{Rescaled Image Feature}

To improve the relationship between task space behavior and image space behavior, it is natural to try to determine an image feature that is as close to the 3-D translation between the camera and the target as possible [28]. Such a choice leads to an interaction matrix close to the identity, leading to a linear and decoupled link between the image features and the translational degrees of freedom. Furthermore, satisfactory behavior of the image features will automatically induce an acceptable behavior in the task space.

We propose to consider a new image feature

$$
\mathbf{f}=F(|\mathbf{q}|) \mathbf{q}_{0}, \quad \text { with } F(|\mathbf{q}|)=\frac{R|\mathbf{q}|}{\sqrt{n^{2}-|\mathbf{q}|^{2}}}
$$

where $\mathbf{q}_{0}=\frac{\mathbf{q}}{|\mathbf{q}|}$ is the normalized first-order moment and $F(|\mathbf{q}|)$ represents a rough approximation of the actual depth $Z$ from the geometric center of the target. $n$ is the number of points observed and $R$ is the approximate radius of the target.

The error $\boldsymbol{\delta}_{\mathrm{f}}$ is defined as follows

$$
\boldsymbol{\delta}_{\mathbf{f}}=\mathbf{f}-\mathbf{f}^{*}=F(|\mathbf{q}|) \mathbf{q}_{0}-F\left(\left|\mathbf{q}^{*}\right|\right) \mathbf{q}_{0}^{*} .
$$

It can be shown that $\dot{\boldsymbol{\delta}}_{\mathrm{f}}=-\boldsymbol{\omega} \times \boldsymbol{\delta}_{\mathrm{f}}-\mathbf{M Q} \boldsymbol{v}$ where $\mathbf{M}(\mathbf{q})=$ $\frac{\partial F(|\mathbf{q}|)}{\partial|\mathbf{q}|} \mathbf{q}_{0} \mathbf{q}_{0}^{\top}+\frac{F(|\mathbf{q}|)}{|\mathbf{q}|}\left(\mathbf{I}_{3}-\mathbf{q}_{0} \mathbf{q}_{0}^{\top}\right)[6]$.

It can be shown that $\mathbf{f} \simeq-\boldsymbol{\xi}$ (where $\boldsymbol{\xi}$ represents the camera position with respect to the target, expressed in the camera frame) and $\mathrm{MQM} \simeq$ $\mathbf{Q}^{-1}[6]$. Since $\mathbf{f} \simeq-\boldsymbol{\xi}$, an intuitive idea is to choose

$$
\boldsymbol{v}=k_{\mathrm{f}} \boldsymbol{\delta}_{\mathrm{f}}, \quad k_{\mathrm{f}}>0 .
$$

Since $\mathbf{M Q} \simeq \mathbf{I}_{3}$, we obtain approximately the same convergence rate for the components of the error [6].

As we will see in Section V-C2, the experimental results using this control law show excellent performance. Its advantage is also that it is easily implemented, since the control law is a direct function of the visual error $\boldsymbol{\delta}_{\mathbf{f}}$. Furthermore, since (10) has the additional passivity property, it is expected to be well-adapted for a wide range of aerial vehicles and experimental conditions.

However, similar to the perspective moments control design, the global asymptotic stability has not been demonstrated.

\section{E. GAS Control Law With Modified Rescaled Image Feature}

In this section, we attempt to define a new image feature and control law that combine the properties of good transient behavior, good local exponential stability, and global asymptotic stability. The approach taken is to define a new scaling function $G(|\mathbf{q}|)$ and scaled image feature

$$
\mathbf{g}=G(|\mathbf{q}|) \mathbf{q}_{0}, \quad \text { with } \dot{\mathrm{g}}=-\boldsymbol{\omega} \times \mathbf{g}-\mathbf{H Q} \boldsymbol{v}
$$

where $G(|\mathbf{q}|)$ can be chosen so that $\mathbf{H}$ induces good properties for asymptotic stability of the resulting control law. Similar to Section III-D for the derivation of $\mathbf{M}$, we have the relationship between matrix $\mathbf{H}$ and function $G(|\mathbf{q}|)[6]: \mathbf{H}(\mathbf{q})=\frac{\partial G(|\mathbf{q}|)}{\partial|\mathbf{q}|} \mathbf{q}_{0} \mathbf{q}_{0}^{\top}+\frac{G(|\mathbf{q}|)}{|\mathbf{q}|}\left(\mathbf{I}_{3}-\mathbf{q}_{0} \mathbf{q}_{0}^{\top}\right)$.

The error $\boldsymbol{\delta}_{\mathrm{g}}$ is defined as follows

$$
\boldsymbol{\delta}_{\mathrm{g}}=\mathbf{g}-\mathbf{g}^{*}=G(|\mathbf{q}|) \mathbf{q}_{0}-G\left(\left|\mathbf{q}^{*}\right|\right) \mathbf{q}_{0}^{*} .
$$

Recalling (12), the dynamics of this error function is given by $\dot{\delta}_{\mathrm{g}}=-\boldsymbol{\omega} \times \boldsymbol{\delta}_{\mathrm{g}}-\mathbf{H Q} \boldsymbol{v}$, and we can note that $\boldsymbol{\delta}_{\mathrm{g}}$ ensures the passivity property, as expected from the choice of $\mathbf{g}$.

Choosing the scale factor $G(|\mathbf{q}|)=\alpha(|\mathbf{q}|) \sqrt{|\mathbf{q}| F(|\mathbf{q}|)}$, and control law

$$
\boldsymbol{v}=k_{\mathrm{g}} \frac{\mathbf{H}(\mathbf{q})}{\alpha(|\mathbf{q}|)^{2}} \boldsymbol{\delta}_{\mathrm{g}}, \quad k_{\mathrm{g}}>0
$$

where $\alpha(|\mathbf{q}|)$ is such that $\alpha\left(\left|\mathbf{q}^{*}\right|\right)=1$ [6], ensures GAS and good local exponential stability of the closed-loop system [6]. The new image feature $\mathbf{g}=G(|\mathbf{q}|) \mathbf{q}_{0}$, and the previous feature $\mathbf{f}=F(|\mathbf{q}|) \mathbf{q}_{0}$ are designed in the same manner: the direction of the feature is given by $\mathbf{q}_{0}$, and the norm is given by the scaling factor $G(|\mathbf{q}|)$ and $F(|\mathbf{q}|)$, 
TABLE I

PROPERTIES OF THE DIFFERENT CONTROL SCHEMES CONSIDERED

\begin{tabular}{|c|l|l|c|c|c|c|c|}
\hline & Section & Control law & GAS & BLES & TC & ALR & P \\
\hline 1. & II & $\boldsymbol{v}=\lambda \mathbf{e}$ & $\times$ & $\sqrt{ }$ & good & $\sqrt{ }$ & $\times$ \\
\hline 2. & III-B & $\boldsymbol{v}=k \boldsymbol{\delta}$ & $\sqrt{ }$ & $\times$ & bad & $\times$ & $\sqrt{ }$ \\
\hline 3. & III-C & $\boldsymbol{v}=k_{\mathbf{A}} \mathbf{A}\left(\mathbf{q}_{0}^{*}\right)^{\top} \boldsymbol{\delta}_{\mathbf{A}}$ & $\sqrt{ }$ & $\sqrt{ }$ & poor & $\times$ & $\sqrt{ }$ \\
\hline 4. & III-D & $\boldsymbol{v}=k_{\mathbf{f}} \boldsymbol{\delta}_{\mathbf{f}}$ & $\times$ & $\sqrt{ }$ & good & $\sqrt{ }$ & $\sqrt{ }$ \\
\hline 5. & III-E & $\boldsymbol{v}=k_{\mathbf{g}} \frac{\mathbf{H}(\mathbf{q})}{\alpha(|\mathbf{q}|)^{2}} \boldsymbol{\delta}_{\mathbf{g}}$ & $\sqrt{ }$ & $\sqrt{ }$ & $\operatorname{good}$ & $\times$ & $\sqrt{ }$ \\
\hline
\end{tabular}

Definitions of the acronyms used are as follows:

GAS: global asymptotic stability, BLES: balanced local exponential stability, TC: transient conditioning, ALR: approximately linear relationship between task space and image space, P: passivity.

respectively. $G(|\mathbf{q}|)$ provides a less aggressive scaling correction than $F(|\mathbf{q}|)[6]$. This improves the sensitivity of the image feature to pixel noise and improves robustness of the closed-loop system. A disadvantage of the new image feature $\mathrm{g}$ is that it is not as closely linked to the actual task space coordinates as the feature $f$ (or the 2-D perspective moments used in Section II). Since $F(|\mathbf{q}|)$ is an approximation of the depth, the feature $\mathbf{f}=F(|\mathbf{q}|) \mathbf{q}_{0}$ is directly related to the 3-D position. In case of the feature $\mathrm{g}$, using the scale factor $G(|\mathbf{q}|)=\alpha(|\mathbf{q}|) \sqrt{|\mathbf{q}| F(|\mathbf{q}|)}$, the relationship between image space and task space is nonlinear. This leads to some degradation of the global transient behavior for certain initial conditions. However, this issue has limited effect on the observed performance of the closed-loop system in practice. As shown in Section V-C3, the practical results are excellent.

\section{ANALYSIS}

A range of IBVS schemes has been presented in Sections II and III. Table I gives summary of the properties for each control scheme in terms of stability, transient behavior, linearity, and passivity.

In practice, two of the most important properties are good transient conditioning (direct convergence of all elements of position in task space without any observed divergence or peaking transients), and balanced local exponential stability (equal asymptotic rate of convergence in all axes of the position in task space). Three control schemes present interesting properties: the perspective image moments (control scheme 1), the rescaled proportional feedback (control scheme 4), and the modified rescaled control (control scheme 5). Among these three best control laws, each one has advantages and drawbacks, and no one is globally better than the others. In the next section, these three control laws are validated and compared through experimental results.

\section{EXPERIMENTAL RESULTS AND COMPARISON OF SOME CONTROL LAWS}

In this section, we provide experimental verification of the performance of the proposed control schemes on an aerial robotic vehicle. The experiments were undertaken on a quadrotor aerial vehicle. The task considered is to stabilize the vehicle with respect to a specified target.

\section{A. Experimental Conditions}

1) Prototype Description: The unmanned aerial vehicle used for the experimentation is a quadrotor, which is an omnidirectional vertical take off and landing (VTOL) vehicle ideally suited for stationary and quasi-stationary flight conditions. It consists of four fixed-pitch propellers linked to an electrical motor at each extremity of a cross frame (see Fig. 2). The vehicle is equipped with an avionics stack including an inertial measurement unit (IMU) supplying the vehicle attitude and a controller board [15]. The embedded loop allowing the attitude stabilization runs at $166 \mathrm{~Hz}$ and the time to reach an attitude order is about $300 \mathrm{~ms}$. A numerical wireless link allows the transmission

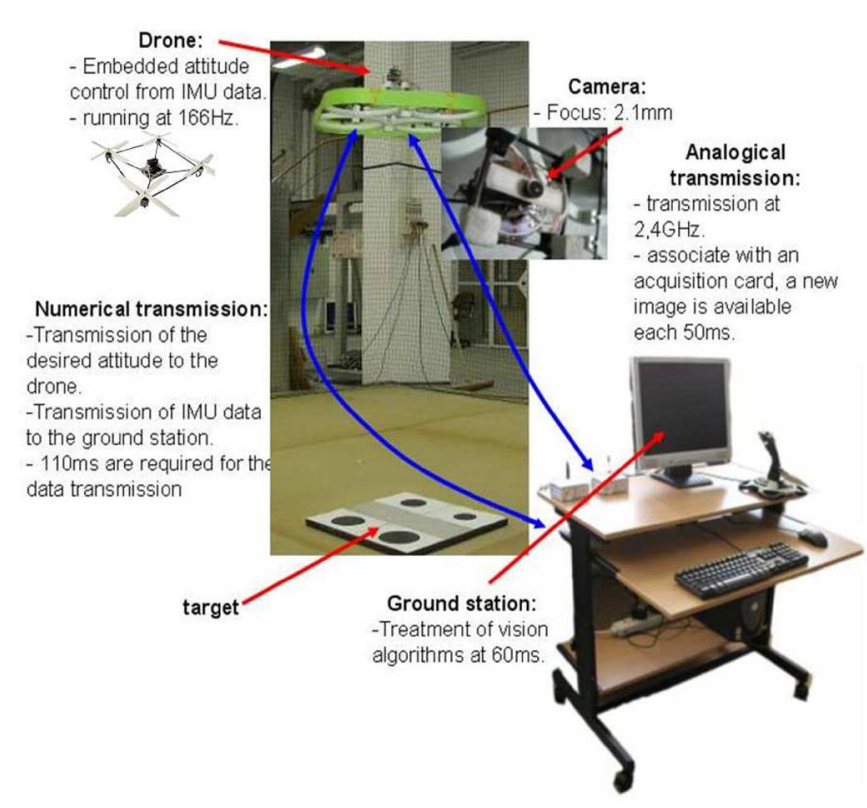

Fig. 2. Experimental system.

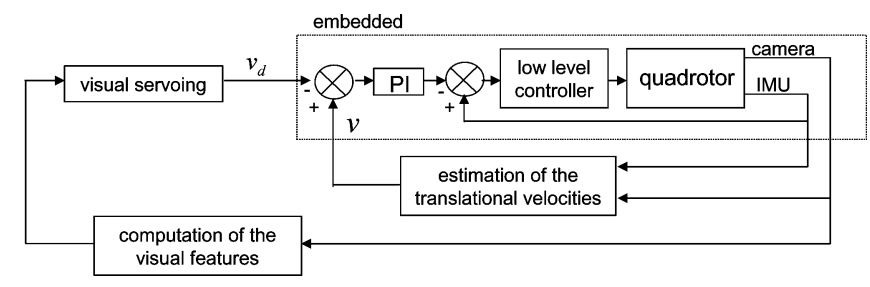

Fig. 3. Low- and high-level control loops.

of the attitude command between the quadrotor and a ground station (Pentium 4) with a time transmission of $110 \mathrm{~ms}$. A camera situated below the quadrotor is embedded and observes a target on the ground, consisting of four black marks on the vertices of a planar rectangle $(30 \mathrm{~cm} \times 40 \mathrm{~cm}$ ) (see Fig. 2). A wireless analog link transmits camera images to the ground station. All the visual servo controls tested are implemented on the ground station at the sample time of $60 \mathrm{~ms}$. Consequently, considering the high sampling rate low level and the low sampling rate high level, we can assume that the low level and the high level control are entirely decoupled. A demonstration based on singular perturbations and similar arguments as in [13] can show the stability of the entire closed-loop system. A 3-D estimation of the vehicle position with respect to the target is also obtained by fusing the data of the embedded IMU and the visual data in a particle filter [3]. This estimate is used to provide an estimate of ground truth for the 3-D behavior of the vehicle and to provide an estimate of the linear velocity of the vehicle that is used by the inner loop controller of the airframe dynamics [14] (see Fig. 3). In this paper, only 2-D visual information is used in the outer IBVS control loop for position regulation.

2) Experimental Protocol: In order to compare the proposed different kinematic visual servo controllers, the initial conditions of the experiments were chosen identically. For each experiment, the quadrotor was servo-controlled to a specific initial position using a standard state-space controller deriving information from the task space position estimate. When the vehicle is stabilized at this position, the visual control is initiated and the 3-D position, obtained from the particle filter, is recorded. This protocol ensures that the flight conditions are 

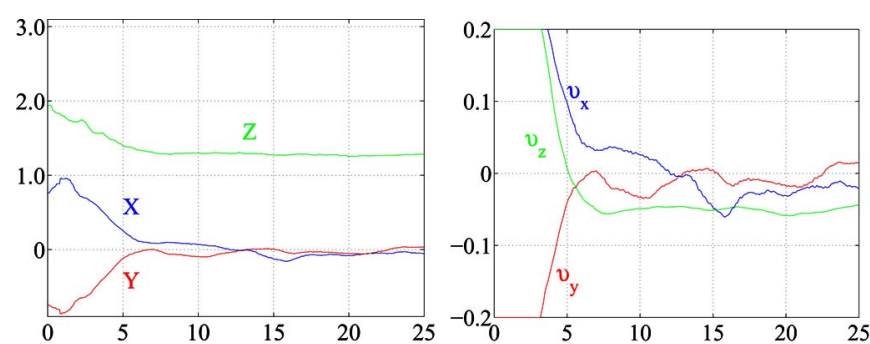

(a)

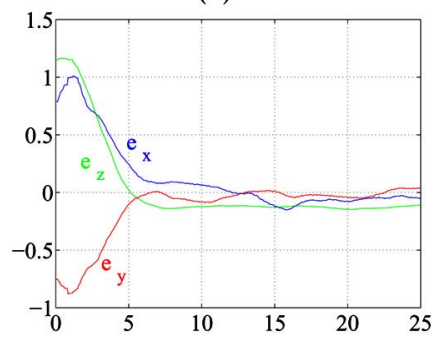

(c)

(d)

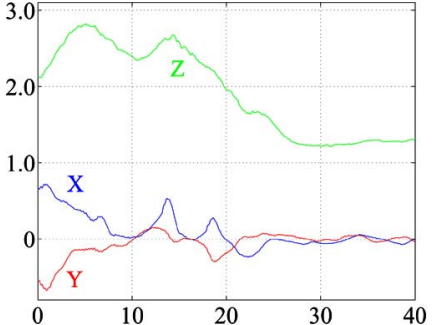

(a)

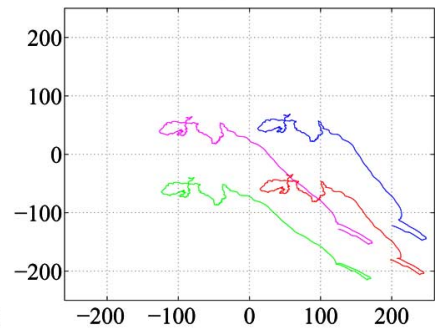

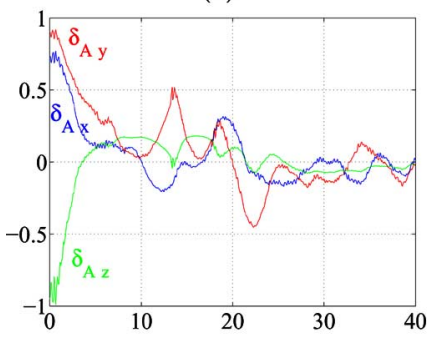

(c)

Fig. 4. Results obtained for $\boldsymbol{v}=0.4 \mathrm{e}$ : time evolution (in seconds) of the real position in the task space (in meters), (a) of them velocity output of the visual servo control $\boldsymbol{v}$ (in meters per seconds) (b). The evolution of the visual error is plotted on (c), and the trajectory of the four black marks in the image plane are plotted in (d).

the same and allows the comparison between the different controllers. The velocity demand was also saturated at $20 \mathrm{~cm} / \mathrm{s}$ to ensure the vehicle remains in quasi-stationary flight regime [16]. Considering times latency and the high sampling of the high-level controller, only low gains have been used. The technique used to tune these gains consists in increasing the gain to increase the bandwidth and stopping just before the UAV becomes unstable. Then, these gains have been reduced in order to have an exponential convergence in about $10 \mathrm{~s}$.

The initial position of the vehicle is $X \simeq 0.7 \mathrm{~m}, Y \simeq-0.65 \mathrm{~m}$, $Z \simeq 2 \mathrm{~m}$, and its desired position is $X \simeq 0 \mathrm{~m}, Y \simeq 0 \mathrm{~m}, Z \simeq 1.4 \mathrm{~m}$ (which is above the center of the target at $1.4 \mathrm{~m}$ height of the ground). The asymptotic value for the matrix $\mathbf{Q}$ is $\mathbf{Q}^{*}=\operatorname{diag}(2.35,2.36,0.057)$ and we have $\mathbf{b} \simeq(0,0,3.96)$.

In the following subsections, four kinematic image-based control schemes for the translational motion of the quadrotor are considered. For each experiment, the 3-D position of the camera in the task space reference frame is depicted, along with the velocity output of the visual servo control law. The evolution of the visual error considered is also depicted, as well as the trajectory of the four black marks in the image plane.

\section{B. Perspective Image Moments}

The classical perspective image moments controller (control law 1) provides a linear correspondence between the image space and task space as long as the relative rotation between image and target plane is small. The resulting closed-loop system response is expected to be satisfactory both in transient performance and asymptotic convergence and in both image and task space. The practical results using the quadrotor are very satisfactory (see Fig. 4) under the considered experimental conditions. However, as a consequence of the limiting assumptions on the rotation, the system is neither GAS nor passive. Moreover, it is expected that strong rotational motion will significantly disturb the performance of the system.

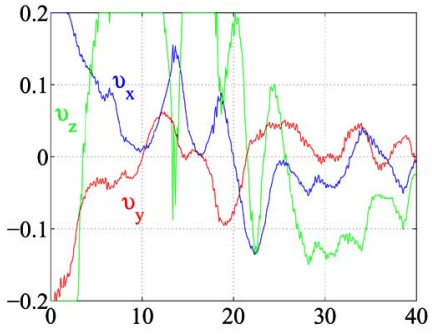

(b)

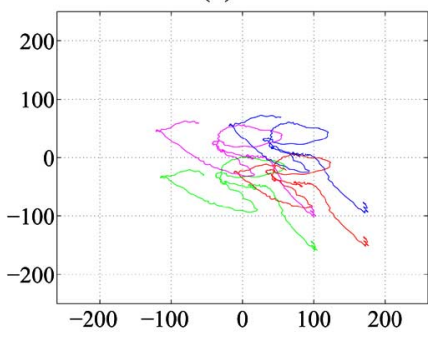

(d)
Fig. 5. Results obtained for $\boldsymbol{v}=k_{\mathbf{A}} \mathbf{A}\left(\mathbf{q}_{0}^{*}\right)^{\top} \boldsymbol{\delta}_{\mathbf{A}}$, configured as Fig. 4.

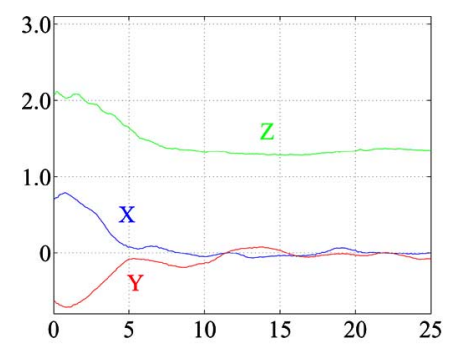

(a)

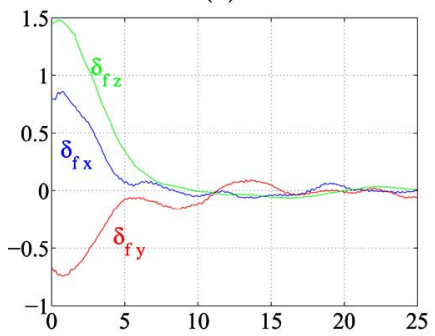

(c)

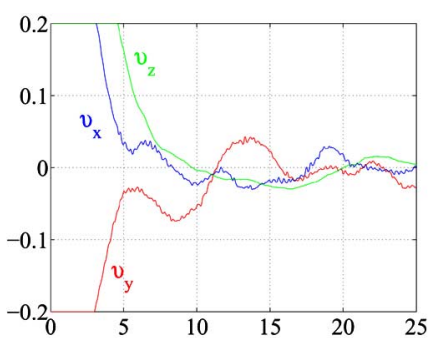

(b)

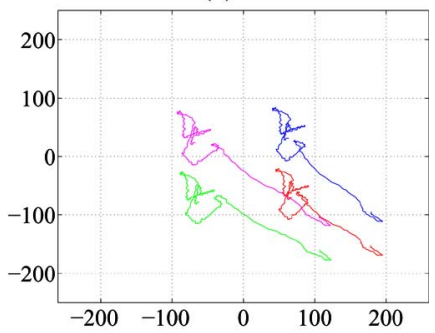

(d)
Fig. 6. Results obtained for $\boldsymbol{v}=0.47 \boldsymbol{\delta}_{\mathrm{f}}$, configured as Fig. 4 .

\section{Spherical Image Moments}

1) Partitioned Control: With the partitioned control law using decomposition at the set point and spherical image moments (control law 3 ), the visual error components are quite perturbed but converge [see Fig. 5(c)]. The problem is that the control law is not adequately far from the desired position. Consequently, we can see that the convergence rate is not the same on the three components of the position, and $Z$ component is not suitable [see Fig. 5(a)]. Moreover, the velocity output of the visual servo control is very disturbed, even after convergence [ $t>25 \mathrm{~s}$, see Fig. 5(b)].

2) Proportional Feedback: The rescaled proportional feedback using spherical image moments (control law 4) has the same desirable image feature properties as control law 1 . The practical results are very satisfactory (see Fig. 6) and similar to the results obtained with control law 1 . 


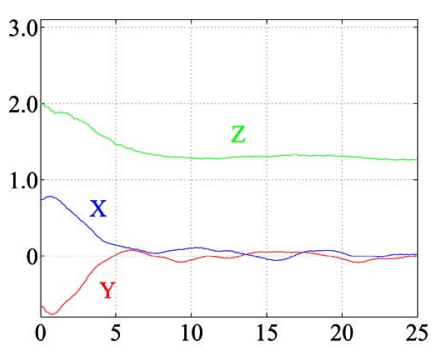

(a)

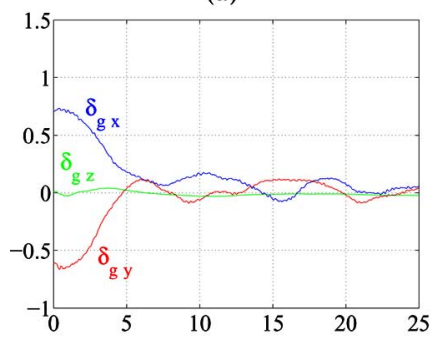

(c)

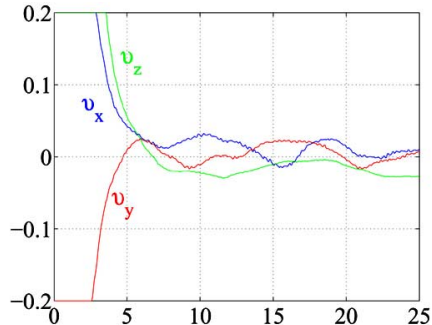

(b)

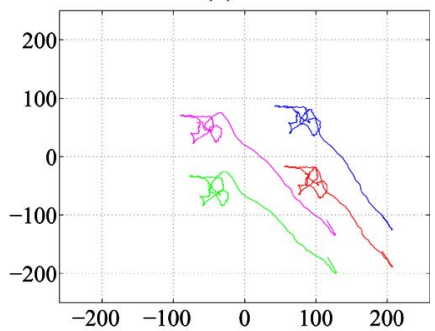

(d)
Fig. 7. Results obtained for $\boldsymbol{v}=0.3 \frac{\mathbf{H}(\mathbf{q})}{\alpha(|\mathbf{q}|)^{2}} \boldsymbol{\delta}_{\mathrm{g}}$, configured as Fig. 4.

In fact, the rescaled visual feature $\mathbf{f}=F(|\mathbf{q}|) \mathbf{q}_{0}$ is very close to the 3 -D position, analogously to the visual features used in control law 1 . The control laws are a simple proportional feedback in the two cases. The advantage of the spherical image moments is that they ensure the passivity property, and should be more robust to aggressive maneuvers of an aerial vehicle as well as leading more naturally to a full dynamic IBVS control design. A potential problem, however, is the requirement to estimate the camera attitude in order to reconstruct the image-based error term. There is no formal proof of GAS for control law 4; however, due to the natural structure of the image feature, we expect that the domain of stability for this control law will be sufficiently large so that unstable behavior will not be encountered in practice.

3) GAS Control Law: The last suitable control law (5) is based on a modified rescaled visual feature, in order to ensure GAS. This control law provides the guarantee of GAS that is missing in control law 4. Its only drawback is that the visual feature is no longer linearly related to the 3-D position and this may lead to slightly degraded transient response in task space.

As can be seen in Fig. 7, this control scheme leads to very satisfactory behavior: equal convergence rates of the visual error components, and equal convergence rates in the task space. Moreover, in the considered experiment, the transient behavior is acceptable.

\section{Noise Sensitivity}

At first glance, the results (see Figs. 4, 6, and 7) for the three suitable control schemes are very similar.

A potential problem with the control laws 4 and 5 is that the rotation matrix $\mathbf{R}$ between the camera frame and the inertial frame has to be estimated. However, this estimation does not seem to introduce noise, delay, or any significant perturbations in practice (compare Fig. 4 with Figs. 6 and 7).

To better understand the noise sensitivity of each control scheme, we have computed the root-mean-square error (RMSE) of the velocity demand over the period between 10 and $25 \mathrm{~s}$, during which period all three closed-loop systems are stabilized in a neighborhood of the set point. Note that to get rid of the effect of the gains, we consider the velocity output without the gains $\lambda, k_{\mathrm{f}}$, and $k_{\mathrm{g}}$. We compute
TABLE II

RMSE OF THE VELOCITIES FOR EACH CONTROL LAW

\begin{tabular}{|l||c||c|c|c|}
\hline & $\sigma_{\boldsymbol{v}}$ & $\sigma_{v_{X}}$ & $\sigma_{v_{Y}}$ & $\sigma_{v_{Z}}$ \\
\hline $\boldsymbol{v}=\mathbf{e}$ & 0.95 & 0.79 & 0.51 & 0.15 \\
\hline $\boldsymbol{v}=\boldsymbol{\delta}_{\mathbf{f}}$ & 1.02 & 0.51 & 0.74 & 0.49 \\
\hline $\boldsymbol{v}=\frac{\mathbf{H}}{\alpha^{2}} \boldsymbol{\delta}_{\mathbf{g}}$ & 1.01 & 0.65 & 0.61 & 0.47 \\
\hline
\end{tabular}

$\sigma_{\boldsymbol{v}}=\sqrt{\sigma_{v_{X}}^{2}+\sigma_{v_{Y}}^{2}+\sigma_{v_{Z}}^{2}}$ along with $\sigma_{v_{K}}=\sqrt{\sum_{i}\left(v_{K_{i}}-\bar{v}_{K}\right)^{2}}$, for $K \in\{X, Y, Z\}$ and where $\bar{v}_{K}$ is the average of $v_{K}$ between 10 and $25 \mathrm{~s}$.

As can be seen in Table II, the noise measured at the output of all control laws is very similar. The three control laws have very similar behavior with respect to noise.

\section{CONCLUSION}

This paper has investigated a suite of image-based kinematic visual servo control schemes to control a quadrotor. Using the well-known perspective image moments to design a classical IBVS translational control law leads to good system behavior in the experimental studies undertaken. However, this control scheme does not ensure global asymptotic stability or passivity of the closed-loop system, both properties that we believe will be important for the development of fully dynamic IBVS control schemes in the future. First-order spherical image moments along with an inertial goal vector allow us to design translational control laws independent from the rotation motion. Global asymptotic stability is obtained by using these visual features and a simple proportional feedback, but the behavior on the $Z$-axis is not acceptable. A range of control laws has been proposed in order to improve the behavior of the system. The most promising approach investigated involves rescaling the spherical image moments to obtain an image feature that minimizes the sensitivity in the depth axis. The perspective image moments control design, as well as three of the control laws using spherical image moments were implemented on the quadrotor. In practice and as expected, three control algorithms lead to acceptable behavior of the system.

\section{REFERENCES}

[1] E. Altuğ, J. P. Ostrowski, and C. J. Taylor, "Control of a quadrotor helicopter using dual camera visual feedback," Int. J. Robot. Res., vol. 24, no. 5, pp. 329-341, 2005.

[2] O. Amidi, T. Kanade, and K. Fujita, "A visual odometer for autonomous helicopter flight," J. Robot. Auton. Syst., vol. 28, pp. 185-193, Aug. 1999.

[3] S. Arulampalam, S. Maskell, N. J. Gordon, and T. Clapp, "A tutorial on particle filters for on-line non-linear/non-gaussian Bayesian tracking," IEEE Trans. Signal Process., vol. 50, no. 2, pp. 174-188, Feb. 2002.

[4] J. R. Azinheira, P. Rives, J. R. H. Carvalho, G. F. Silveira, E. C. de Paiva, and S. S. Bueno, "Visual servo control for the hovering of an outdoor robotic airship," in Proc. IEEE Int. Conf. Robot. Autom., Washington, DC, May 2002, vol. 3, pp. 2787-2792.

[5] O. Bourquardez and F. Chaumette, "Visual servoing of an airplane for auto-landing," in IEEE/RSJ Int. Conf. Intell. Robots Syst., IROS 2007, San Diego, CA, Oct., pp. 1314-1319.

[6] O. Bourquardez, R. Mahony, N. Guenard, F. Chaumette, T. Hamel, and L. Eck. (2007, Jul.). Kinematic visual servo control of a quadrotor aerial vehicle. IRISA, Rennes, France, Tech. Rep. 1858 [Online]. Available: http://www.irisa.fr/lagadic/publi/year/2007-fra.html

[7] F. Chaumette and S. Hutchinson, "Visual servo control, Part I: Basic approaches," IEEE Robot. Autom. Mag., vol. 13, no. 4, pp. 82-90, Dec. 2006.

[8] P. Corke and S. A. Hutchinson, "A new partitioned approach to imagebased visual servo control," IEEE Trans. Robot. Autom., vol. 17, no. 4, pp. 507-515, Aug. 2001. 
[9] P. I. Corke and M. C. Good, "Dynamic effects in visual closed-loop systems," IEEE Trans. Robot. Autom., vol. 12, no. 5, pp. 671-683, Oct. 1996.

[10] B. Espiau, F. Chaumette, and P. Rives, "A new approach to visual servoing in robotics," IEEE Trans. Robot. Autom., vol. 8, no. 3, pp. 313-326, Jun. 1992

[11] E. Frazzoli, M. A. Dahleh, and E. Feron, "Real-time motion planning for agile autonomous vehicles," J. Guid. Control Dyn., vol. 25, no. 1, pp. 116-129, 2002.

[12] J. Gangloff and M. de Mathelin, "Visual servoing of a 6 DOF manipulator for unknown 3D profile following," IEEE Trans. Robot. Autom., vol. 18, no. 4, pp. 511-520, Aug. 2002.

[13] N. Guenard, "Optimisation et implémentation de lois de commande embarquées pour la téléopération intuitive de micro drones aériens X4-Flyer," Ph.D. dissertation, Université de Nice Sophia Antipolis, Nice, France, 2007.

[14] N. Guenard, T. Hamel, and L. Eck, "Control law for the tele operation of an unmanned aerial vehicle known as an X4-flyer," in Proc. IEEE/RSJ Int. Conf. Intell. Robots Syst., Beijing, China, Oct. 2006, pp. 3249-3254.

[15] N. Guenard, T. Hamel, and R. Mahony, "A practical visual servo control for a unmanned aerial vehicle," IEEE Trans. Robot., vol. 24, no. 2, pp. 331-340, Apr. 2008.

[16] N. Guenard, T. Hamel, V. Moreau, and R. Mahony, "Design of a controller allowed the intuitive control of an X4-flyer," presented at the Int. IFAC Symp. Robot Control, Unive. Bologna, Bologna, Italy, Sep. 2006.

[17] T. Hamel and R. Mahony, "Visual servoing of an under actuated dynamic rigid-body system: An image based approach," IEEE Trans. Robot. Autom., vol. 18, no. 2, pp. 187-198, Apr. 2002.

[18] T. Hamel and R. Mahony, "Image based visual servo-control for a class of aerial robotic systems," Automatica, vol. 43, no. 11, pp. 1975-1983, Nov. 2007.

[19] K. Hatano and K. Hashimoto, "Image-based visual servo using zoom mechanism," in Proc. SICE 2003 Annu. Conf., Aug. 4-6, vol. 3, pp. 24432446.

[20] R. Kelly, R. Carelli, O. Nasisi, B. Kuchen, and F. Reyes, "Stable visual servoing of camera-in-hand robotic systems," IEEE/ASME Trans. Mechatronics, vol. 5, no. 1, pp. 39-48, Mar. 2000.

[21] E. Malis, F. Chaumette, and S. Boudet, "2 1/2 D visual servoing," IEEE Trans. Robot. Autom., vol. 15, no. 2, pp. 238-250, Apr. 1999.

[22] K. Nordberg, P. Doherty, G. Farnebäck, P.-E. Forssén, G. Granlund, A. Moe, and J. Wiklund, "Vision for a UAV helicopter," presented at the IEEE/RSJ Int. Conf. Intell. Robots Syst., Workshop Aerial Robot., Lausanne, Switzerland, Oct. 2002, pp. xxxx.

[23] R. Pissard-Gibollet and P. Rives, "Applying visual servoing techniques to control of a mobile hand-eye system," in Proc. IEEE Int. Conf. Robot. Autom., Nagasaki, Japan, 1995, pp. 166-171.

[24] P. Rives and J. R. Azinheira, "Visual auto-landing of an autonomous aircraft,” INRIA, Sophia Antipolis, France, Tech. Rep. 4606, Nov. 2002.

[25] H. Romero, R. Benosman, and R. Lozano, "Stabilization and location of a four rotor helicopter applying vision," in Proc. Amer. Control Conf., ACC 2006, Minneapolis, MN, Jun., pp. 3930-3936.

[26] S. Saripalli, J. F. Montgomery, and G. S. Sukhatme, "Visually-guided landing of an unmanned aerial vehicle," IEEE Trans. Robot. Autom., vol. 19, no. 3, pp. 371-381, Jun. 2003.

[27] O. Shakernia, Y. Ma, T. Koo, and S. Sastry, "Landing an unmanned air vehicle: Vision based motion estimation and nonlinear control," Asian J. Control, vol. 1, no. 3, pp. 128-145, Sep. 1999.

[28] O. Tahri and F. Chaumette, "Point-based and region-based image moments for visual servoing of planar objects," IEEE Trans. Robot., vol. 1, no. 6, pp. 1116-1127, Dec. 2005.

[29] A. D. Wu, E. N. Johnson, and A. A. Proctor, "Vision-aided inertial navigation for flight control," presented at the AIAA Guid., Navigat., Control Conf. Exhib., San Francisco, CA, Aug. 2005.

[30] H. Zhang and J. P. Ostrowski, "Visual servoing with dynamics: Control of an unmanned blimp," in Proc. IEEE Int. Conf. Robot. Autom., ICRA 1999, Detroit, MI, May, vol. 1999, pp. 618-623.

\section{Qualitative Vision-Based Path Following}

Zhichao Chen and Stanley T. Birchfield, Senior Member, IEEE

Abstract-We present a simple approach for vision-based path following for a mobile robot. Based upon a novel concept called the funnel lane, the coordinates of feature points during the replay phase are compared with those obtained during the teaching phase in order to determine the turning direction. Increased robustness is achieved by coupling the feature coordinates with odometry information. The system requires a single off-the-shelf, forward-looking camera with no calibration (either external or internal, including lens distortion). Implicit calibration of the system is needed only in the form of a single controller gain. The algorithm is qualitative in nature, requiring no map of the environment, no image Jacobian, no homography, no fundamental matrix, and no assumption about a flat ground plane. Experimental results demonstrate the capability of real-time autonomous navigation in both indoor and outdoor environments and on flat, slanted, and rough terrain with dynamic occluding objects for distances of hundreds of meters. We also demonstrate that the same approach works with wide-angle and omnidirectional cameras with only slight modification.

Index Terms-Control, feature tracking, mobile robot navigation, visionbased navigation.

\section{INTRODUCTION}

Route-based knowledge, in which the spatial layout of an environment is recorded from the perspective of a ground-level observer, is an important component of human and animal navigation systems [31]. In this representation, navigating from one location to another involves comparing current visual inputs with a sequence of views captured along the path in a previous instance. Applications that would benefit from such a path-following capability include courier and delivery robots [4], robotic tour guides [32], or reconnaissance robots following a scout [7].

One approach to path following is visual servoing, in which the robot is controlled to align the current image with a reference image, both taken by an onboard camera [14]. Such an approach generally employs a Jacobian to relate the coordinates of world points to their projected image coordinates [5], a homography or fundamental matrix to relate the coordinates between images [20], [27], [29], [36], or bundle adjustment to minimize the reprojection error over multiple image frames [28]. As a result, the camera usually must be calibrated [5], [27], [28], [36], and even uncalibrated systems require lens

Manuscript received January 14, 2008; revised September 20, 2008. First published April 14, 2009; current version published June 5, 2009. This paper was recommended for publication by Associate Editor J. D. Tardos and Editor L. Parker upon evaluation of the reviewers' comments. This work was supported in part by a Ph.D. Fellowship from the National Institute for Medical Informatics.

The authors are with the Department of Electrical and Computer Engineering, Clemson University, Clemson, SC 29634 USA (e-mail: zhichac@clemson.edu; stb@clemson.edu).

This paper has supplementary downloadable multimedia material available at http://ieeexplore.ieee.org, provided by the author. The material includes the video ("ramp.avi") that shows the mobile robot following a predetermined path The four quadrants are as follows: (Top left): Live video from the onboard camera during the replay phase. Red squares indicate features, with green outlines indicating features that guide the robot to the right, and yellow outlines indicating features that guide the robot to the left. (Top right): The milestone image captured during the teaching phase. (Bottom left): The robot as captured by an offboard video camera. (Bottom right): Top-down view of the path traveled by the robot (white: teaching, red: replay). The video is an AVI file that plays under Windows Media Player 11 (2006), as well as earlier versions such as Windows Media Player 6.4 (1999), on Windows XP. The size is 8.35 MB. Additional videos may be found at http://www.ces.clemson.edu/ stb/research/mobile_robot. Contact stb@clemson.edu for further questions about this work.

Digital Object Identifier 10.1109/TRO.2009.2017140 